\title{
Monitoring the Differentiation and Migration Patterns of Neural Cells Derived from Human Embryonic Stem Cells Using a Microfluidic Culture System
}

\author{
Nayeon Lee, ${ }^{1,4}$ Jae Woo Park ${ }^{2,4}$, Hyung Joon Kim², Ju Hun Yeon², Jihye Kwon', Jung Jae Ko', \\ Seung-Hun Oh${ }^{1}$, Hyun Sook Kim ${ }^{1}$, Aeri Kim ${ }^{1}$, Baek Soo Han ${ }^{3}$, Sang Chul Lee ${ }^{3}$, Noo Li Jeon ${ }^{2, *}$, and \\ Jihwan Song ${ }^{1, *}$
}

\begin{abstract}
Microfluidics can provide unique experimental tools to visualize the development of neural structures within a microscale device, which is followed by guidance of neurite growth in the axonal isolation compartment. We utilized microfluidics technology to monitor the differentiation and migration of neural cells derived from human embryonic stem cells (hESCs). We co-cultured hESCs with PA6 stromal cells, and isolated neural rosette-like structures, which subsequently formed neurospheres in suspension culture. Tuj1-positive neural cells, but not nestinpositive neural precursor cells (NPCs), were able to enter the microfluidics grooves (microchannels), suggesting that neural cell-migratory capacity was dependent upon neuronal differentiation stage. We also showed that bundles of axons formed and extended into the microchannels. Taken together, these results demonstrated that microfluidics technology can provide useful tools to study neurite outgrowth and axon guidance of neural cells, which are derived from human embryonic stem cells.
\end{abstract}

\section{INTRODUCTION}

Human embryonic stem cells (hESCs) are derived from the inner cell mass of the preimplantation-stage embryo. These

${ }^{1} \mathrm{CHA}$ Stem Cell Institute, CHA University, Seoul 135-081, Korea, ${ }^{2}$ Division of World Class University Multiscale Mechanical Design, School of Mechanical and Aerospace Engineering, Seoul National University, Seoul 151-742, Korea, ${ }^{3}$ Research Center for Integrated Cellulomics, Korea Research Institute of Bioscience and Biotechnology, Daejeon 305-806, Korea, ${ }^{4}$ These authors contributed equally to this work. *Correspondence: jsong@cha.ac.kr (JS); njeon@snu.ac.kr (NLJ)

Received 26 May, 2014; revised 29 May, 2014; accepted 29 May, 2014; published online 18 June, 2014

Keywords: axons, human embryonic stem cells, microfluidics, migration, neural differentiation cells have the potential to proliferate indefinitely and to differentiate into all cell types found in the body (Reubinoff et al., 2000; Thomson et al., 1998). For this reason, hESCs have been widely regarded as ideal sources for cell replacement therapies to treat various forms of degenerative and metabolic and genetic diseases, including neurological disorders. However, despite extensive studies of hESCs, there is still a lack of understanding of their proliferation and differentiation, on a detailed microscopic level (Blow, 2008). These processes may be influenced by subtle changes of the stem cell microenvironment, including the levels of growth factor secretion and extracellular matrix deposition. Physiological changes, such as temperature and $\mathrm{pH}$, can also influence cellular behavior. For these reasons, it is essential to minimize alterations of various parameters that affect the microenvironment, to better control cellular behavior under normal or various experimental conditions.

Microfluidics provides a new technological platform, based on the flow of liquids in channels of micrometer size (Hamon and Hong 2013; Park et al., 2006; Taylor et al., 2005). This new technology offers useful experimental tools for studying cellular neuroscience, which involves development of neural structures within a microscale device, followed by guidance of neurite growth into the axonal isolation compartment, which can be easily visualized and monitored using time-lapse video microscopy. Compared with conventional culture systems, a microfluidic device can provide a physiologically more relevant cellular environment, by generating fluid flows which can maintain a more constant and soluble microenvironment (Breslauer et al., 2006). This can be achieved by applying gradient materials, which provide a potentially unlimited range of concentrations by using multi-inputs under different experimental conditions (Millet et al., 2010). Recently, this microfluidic technology has been developed to study neural development more precisely, allowing cell bodies and axons to be isolated and monitored differentially (Millet and Gillette, 2012; Park et al., 2009; Taylor et al., 2005), and allowing the study of signaling mechanisms using a microfluidic neuronal culture system (Park et al., 2013; Taylor and Jeon, 2010). 

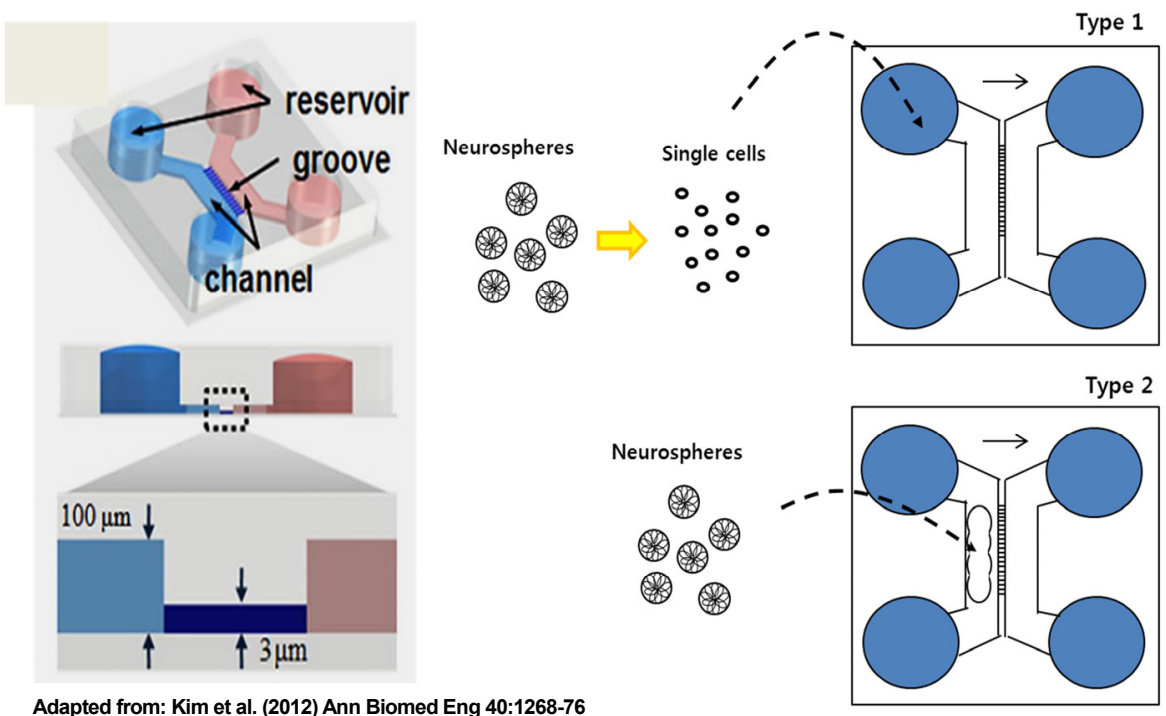

Fig. 1. Neural culture platform of the microfluidic-based chamber system. The Type 1 device was used for neural differentiation from single cells, whereas the Type 2 device was used for neurosphere differentiation into mature neurons.

Adapted from: Kim et al. (2012) Ann Biomed Eng 40:1268-76
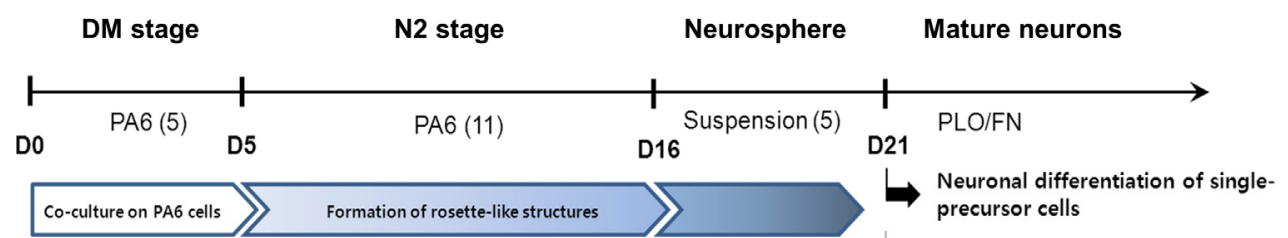

$\Rightarrow$ Differentiation into mature neurons (CNS)

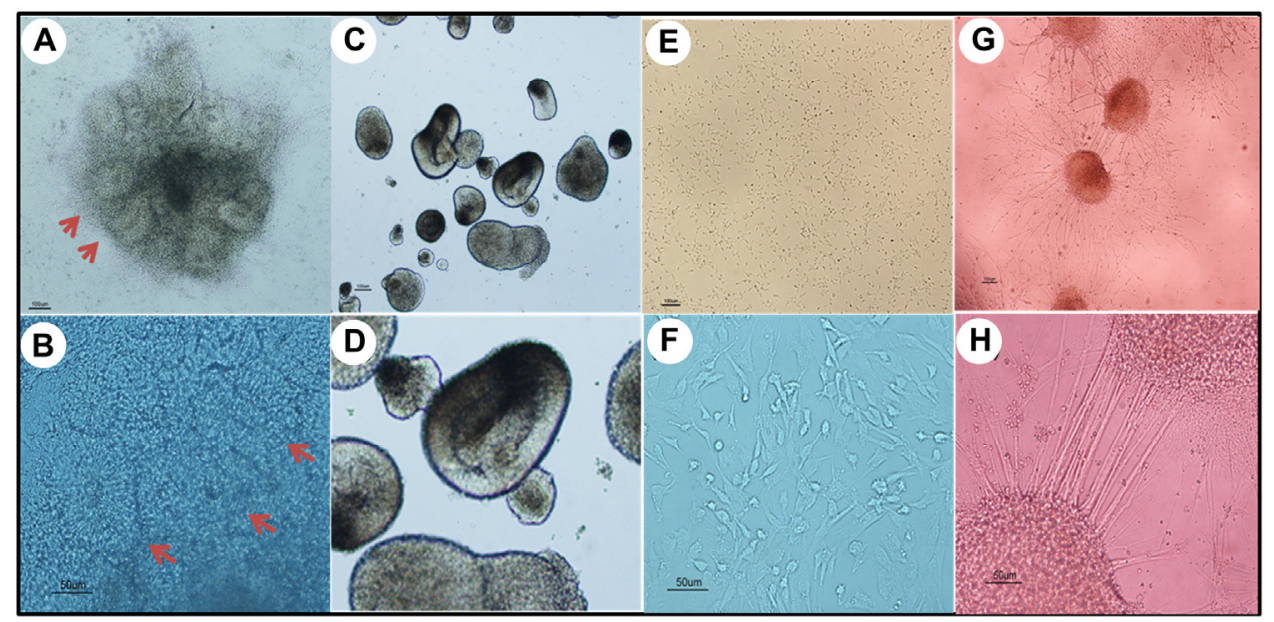

Fig. 2. Schematic diagram for stepwise neural differentiation of hESCs (H9), and their representative morphologies at each stage. Upper panels (A, C, E, and G) show low magnification, with enlarged images in the bottom panels $(B, D, F$, and $H)$, respectively. (A, B) Rosette-like structures formed by co-culturing hESCs with PA6 stromal cells. (C, D) Neurospheres formed in suspension culture. ( $E, F)$ Dissociated neural precursor cells maintained on PLO/FN double-coated dishes. ( $G, H)$ Neurospheres differentiated into mature neurons in N2 medium containing BDNF. Abbreviations: hESCs, human embryonic stem cells; PLO/FN, poly-L-ornithine and fibronectin; BDNF, brain-derived neurotrophic factor.

In this study, we utilized microfluidics technology to monitor the differentiation and migration of neural cells that were derived from hESCs, and found that this technology could be used to study neurite outgrowth and axon guidance under normal and experimental conditions.

\section{MATERIALS AND METHODS}

Preparation of microfluidic devices

Microfluidic devices were prepared as described previously (Kim et al., 2012). Briefly, we fabricated the chambers in polydimethylsiloxane (PDMS) using soft lithography and replica molding. Using photolithography, two layers of negative photoresist, SU-8, on a silicon wafer, were patterned, resulting in a master with positive relief patterns of cell culture compartments and microgrooves (Rhee et al., 2005; Taylor et al., 2003). Next, we cast and cured a prepolymer mixture of Sylgard 184 (Dow Corning, USA) against the positive relief master, to obtain a negative replica-molded piece. After curing, the PDMS was peeled away from the master and reservoirs were punched out with a sharpened 


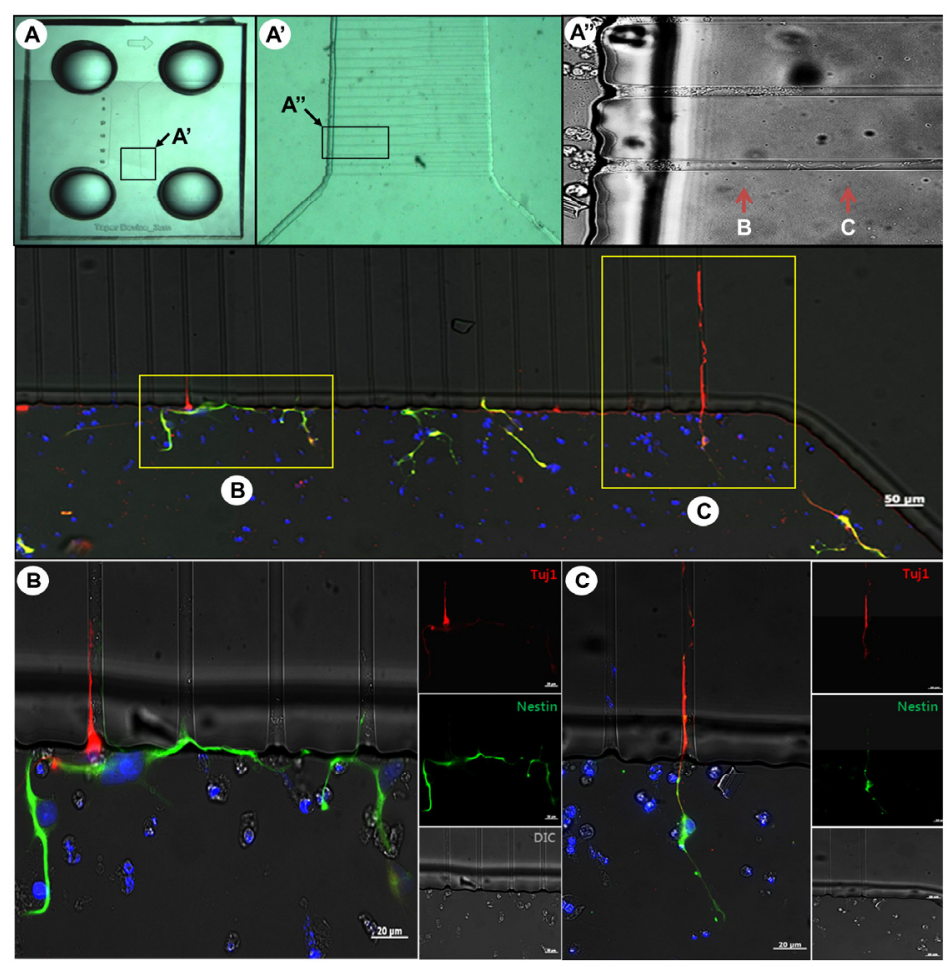

Fig. 3. Outgrowth of neural precursor cells derived from hESCs in the microfluidic device. (A) Overall configuration of the microfluidic device, with enlarged images $\left(A^{\prime}\right.$, $\left.A^{\prime \prime}\right)$ showing the migration of cells. $(B, C)$ Immunocytochemical staining of migrating neurons. A $90^{\circ}$ counterclockwise rotated view of $(A)$ is shown. Tuj1-positive neural cells $(C)$, but not Nestin-positive NPCs, entered the microchannels of the microfluidic device $(B)$. Abbreviation: NPCs, neural precursor cells.

needle. The PDMS pieces were sterilized with $70 \%$ ethanol. Subsequently, cleaned glass coverslips $(24 \times 40 \mathrm{~mm}$, No. 1 , Corning Inc., USA) were immersed in a sterile aqueous solution of $1.0 \mathrm{mg} / \mathrm{ml}$ poly-L-lysine (PLL, M.W. 70,000-150,000; Sigma, USA) in $0.1 \mathrm{M}$ borate buffer, for $24 \mathrm{~h}$ before use.

\section{Human ESC culture and maintenance}

H9 hESCs (obtained from WiCell, USA), were cultured on mitomycin C-treated mouse embryonic fibroblast cells in hESC medium as described previously (Jeon et al., 2012; Lee et al., 2009). The medium was changed daily, and cells were transferred onto new feeder cells every 6 days, using CTK solution, consisting of $0.1 \mathrm{mg} / \mathrm{ml}$ collagenase type IV, $0.25 \%$ trypsin, 1 $\mathrm{mM} \mathrm{CaCl}_{2}$, and $20 \%$ Knockout $^{\mathrm{TM}} \mathrm{SR}$ (all from Gibco). All cultures were maintained at $37^{\circ} \mathrm{C}$, in a $5 \% \mathrm{CO}_{2}$ incubator.

\section{Neuronal differentiation of hESCs}

Neuronal differentiation of $\mathrm{H} 9 \mathrm{hESCs}$ were induced by coculturing with PA6 stromal cells. Briefly, $\mathrm{H} 9$ cells were mechanically dissected into small colonies and transferred onto PA6 stromal cells with differentiation medium (DM), consisting of Glasgow's Minimal Essential Medium (GMEM; Invitrogen, USA), supplemented with $10 \%$ Knockout $^{\mathrm{TM}} \mathrm{SR}, 1 \%$ penicillin, $1 \%$ streptomycin, $1 \%$ nonessential amino acids, and $0.1 \% \beta$ mercaptoethanol. After 4 days, the DM was changed to N2 medium containing an N2 supplement (Gibco), $3 \mathrm{mM}$ Dglucose (Sigma), $0.2 \mathrm{mM}$ ascorbic acid (Sigma), and $2 \mathrm{mM} \mathrm{L-}$ glutamine (Welgene, Korea). Medium was changed every other day. At 16 days, the rosette-like structures were isolated and transferred to Petri dishes, which resulted in the formation of neurospheres in N2 medium supplemented with $10 \mathrm{ng} / \mathrm{ml}$ basic fibroblast growth factor (bFGF). To make neural precursor cells (NPCs), we dissociated neurospheres into single cells using Accutase $^{\mathrm{TM}}$ (Chemicon, USA), for $10 \mathrm{~min}$, at $37^{\circ} \mathrm{C}$, and plated cells onto a device, double-coated with $15 \mu \mathrm{g} / \mathrm{ml}$ poly-Lornithine (PLO; Sigma) and $1 \mu \mathrm{g} / \mathrm{ml}$ fibronectin (FN; Sigma). Neuronal differentiation was also directly induced from neurospheres by reattaching them onto a PLO/FN-coated device. In both cases, to induce mature neuron differentiation, we replaced bFGF with $20 \mathrm{ng} / \mathrm{ml}$ brain-derived neurotrophic factor (BDNF) (R\&D Systems, USA) in the N2 medium.

Monitoring neuronal differentiation and neurite outgrowth To observe neurite outgrowth into grooves, NPCs and neurospheres were directly attached onto PLO/FN-coated microfluidic devices and cultured for 5 to 7 days in the presence of 20 $\mathrm{ng} / \mathrm{ml}$ BDNF. For monitoring of neuronal differentiation and neurite outgrowth, attached neurospheres were placed inside a $\mathrm{CO}_{2}$ incubator mini chamber, and observed using an inverted microscope (Olympus, Japan). Medium was loaded onto each lane of the microfluidic device, every other day. At 7 days after attachment, devices were fixed in $4 \%$ paraformaldehyde, followed by immunocytochemistry.

\section{Immunocytochemistry}

To analyze the marker expression of NPCs or neurospherederived neural cells in microfluidic devices, the devices were fixed in $4 \%$ paraformaldehyde for $15 \mathrm{~min}$ and washed three times in $\mathrm{Ca}^{2+}-, \mathrm{Mg}^{2+}$-containing phosphate-buffered saline (PBS; Welgene, Korea). Primary antibodies used in this study were human Nestin (1:200, Chemicon) and type III $\beta$-tubulin (Tuj1) (1:500, Chemicon). Secondary antibodies used were goat anti-mouse IgG-conjugated Alexa-555 (1:200, Molecular Probes, USA) and goat anti-rabbit IgG-conjugated Alexa-488 (1:200, Molecular Probes). Following the secondary antibody reaction, nuclei of cells were counterstained with DAPI (1:1000, Roche). Subsequently, they were mounted in Vectashield ${ }^{\mathrm{TM}}$ (Vector Laboratories, USA). Stained NPCs and neurons were 


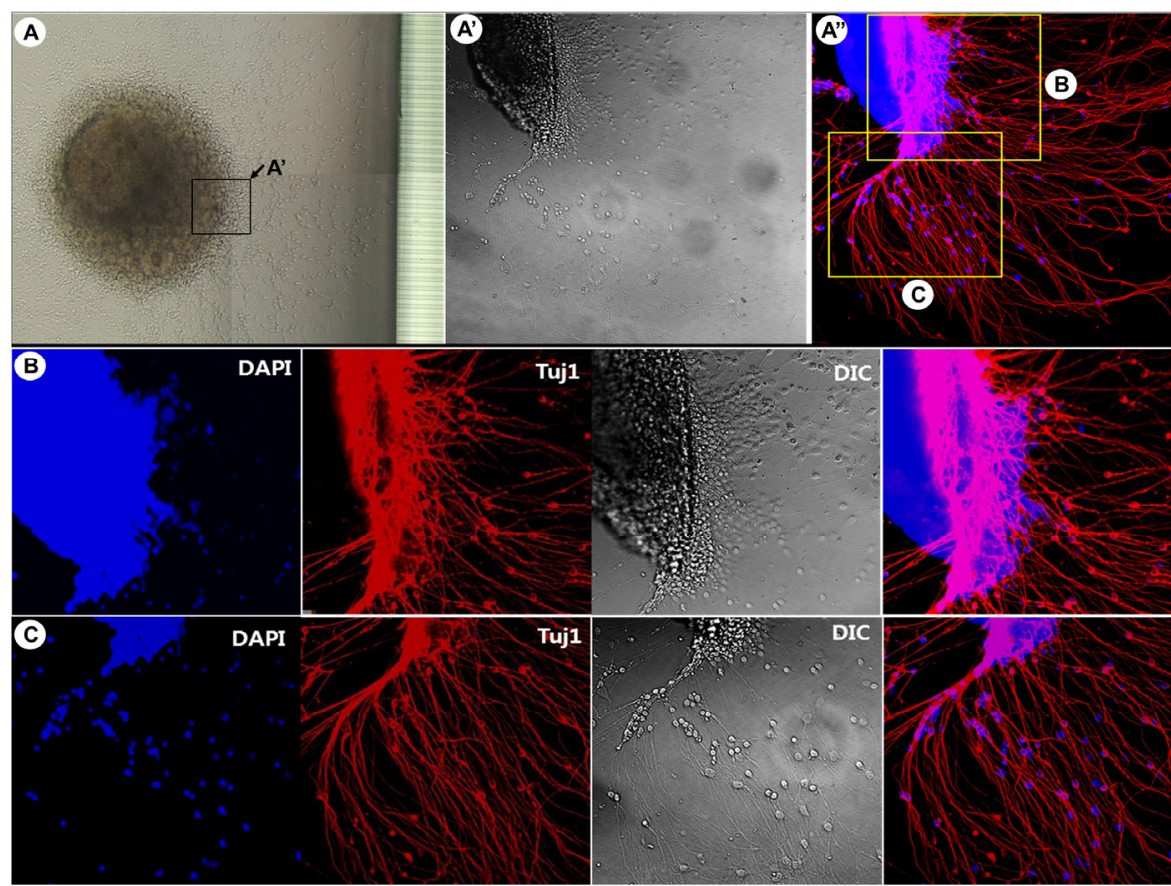

Fig. 4. Differentiation of neurospheres into mature neurons. (A) Overall view of attached and differentiating hESC-derived neurospheres, with higher magnification $\left(A^{\prime}, A^{\prime \prime}\right)$. (B, C) Morphologies of neurons in different regions. The hESC-derived neurospheres differentiated efficiently into mature neurons in microfluidic devices to form neural networks. Abbreviation: hESCs, human embryonic stem cells.

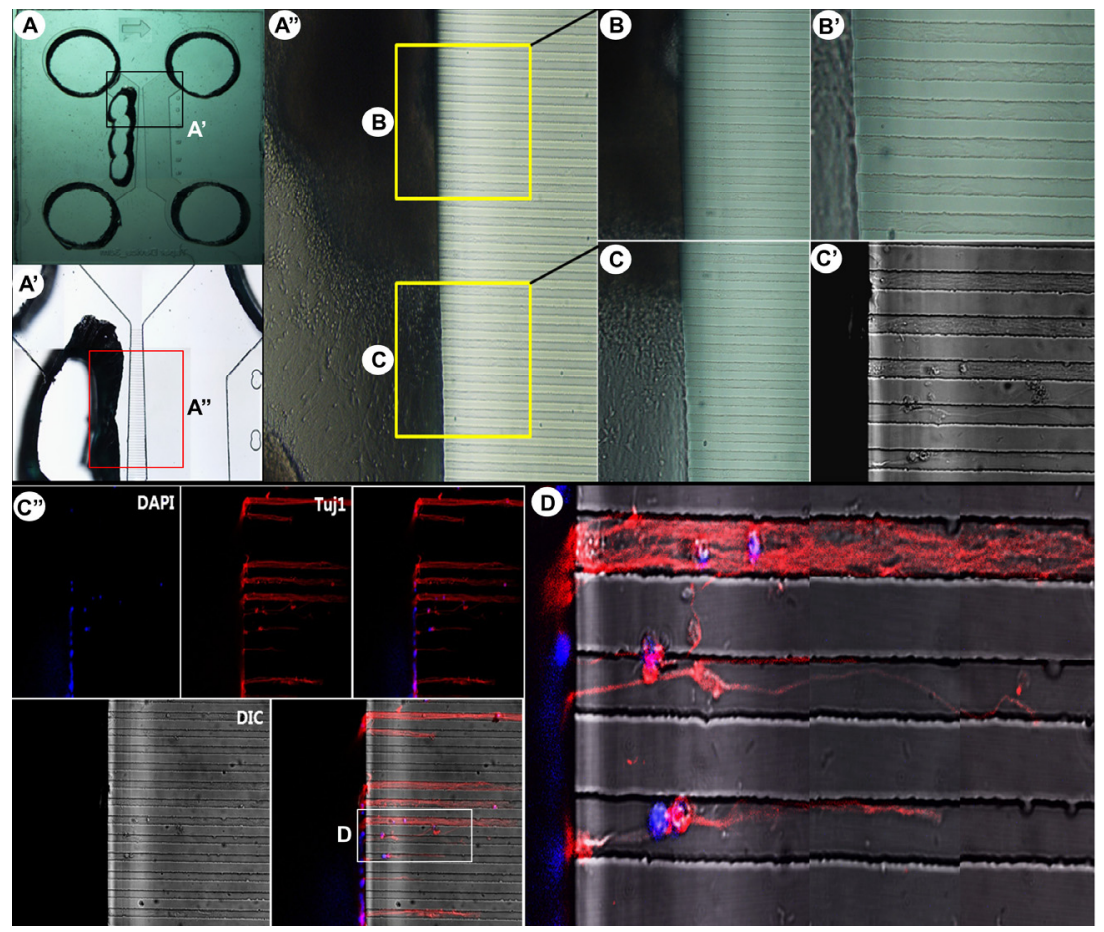

Fig. 5. Growth and migration of an axon bundle through microchannels. (A) Overall view of a Type 2 device, with its enlarged images $\left(A^{\prime}, A^{\prime \prime}\right)$. $(B, C)$ Enlarged images showing the presence and migration of neurons derived from neurospheres, with higher magnification $\left(\mathrm{B}^{\prime}, \mathrm{C}^{\prime}\right)$. Immunocytochemical staining showing the accumulation and migration of a single axon or a bundle of axons through microchannels (C", D). Type III $\beta$-tubulin (Tuj1)-positive neurons are shown in red.

examined and photographed using a confocal laser scanning microscope imaging system (LSM510, Carl Zeiss, Inc., USA).

\section{RESULTS}

Establishment of a neural culture platform using microfluidic-based chamber systems
We have previously established various microfluidic-based chamber systems (Kim et al., 2012). In this study, we used two types of microfluidic devices to monitor neuronal differentiation of hESCs (Fig. 1). The Type 1 device was used for neural differentiation from single cells, whereas the Type 2 device was used for differentiation into mature neurons from neurospheres. 
Step-wise neural differentiation of hESCs

There are currently many protocols to differentiate hESCs into neural lineages. In our laboratory, we established optimal conditions for neuronal differentiation by co-culturing with PA6 stromal cells, followed by rosette isolation and suspension culture to induce neurosphere formation (Jeon et al., 2012; Lee et al., 2009). These neurospheres were then either dissociated into single cells to form neural precursor cells (NPCs), or plated onto PLO/FN double-coated dishes to promote neuronal differentiation. In both cases, N2 medium was supplemented with BDNF in the absence of bFGF to differentiate NPCs or neurospheres into mature neurons (Fig. 2). For microfluidic experiments, either NPCs or neurospheres were attached or loaded onto the device. Figure 2 (bottom panels) shows representative morphologies at each stage during the step-wise neuronal differentiation of human ES cells.

Migration of hESC-derived NPCs in the microfluidic device To make NPCs, neurospheres were first dissociated into single cells. They were then allowed to attach onto a PLO/FN-coated Type 1 device (Fig. 1) at a density of $5 \times 10^{3}$ cells $/ \mathrm{cm}^{2}$. After 5 to 7 days, NPCs were fixed for immunocytochemical analysis. Tuj1-positive neural cells, but not Nestin-positive NPCs, were able to enter the microchannels of the microfluidic device (Figs. $3 \mathrm{~B}$ and $3 \mathrm{C}$ ), indicating that the migration capacity of neural cells was dependent on the stage of neuronal differentiation. Because this type of microfluidic system allowed preferential migration of axons into the channels (Kim et al., 2012), it was likely that Nestin-positive cells (Fig. 3B, green) which did not reach the channels, would represent either immature NPCs or dendrites, whereas Tuj1-positive cells (Fig. 3C, red), which migrated into the channels, would be axons. Antibodies to neurofilmanent NF-H or Tau could then be used to identify axons, and MAP2 antibody used to identify dendrites (Kosik and Finch, 1987).

Neurite outgrowth and migration of hESC-derived neurospheres in the microfluidic device

To induce neuronal differentiation of hESC-derived neurospheres, cells were attached to a PLO/FN-coated Type 2 device (Fig. 1), in differentiation medium containing BDNF, which promotes neuronal differentiation (Cohen et al., 2011). Figure $4 \mathrm{~A}$ shows representative morphological features of neurospheres following attachment, with extensive neurite outgrowth at the periphery of neurospheres. Immunocytochemical analysis using confocal microscopy demonstrated that the differentiated cells consisted of mature neurons (Figs. 4B and 4C). Together, these results demonstrated that hESC-derived neurospheres can efficiently differentiate into mature neurons and form neural networks in a microfluidic device. We also examined the detailed growth and migration patterns of neurons derived from neurospheres in the microfluidic device (Fig. 5). Tuj1-positive neurons appeared inside the microchannels of the device. This device was specifically designed for guidance of axons by controlling the length of microchannels, thereby only axons from cell bodies could grow and move into the axon compartment (Kim et al., 2012). In principle, both axons and dendrites could grow through the axon-guiding channels, but dendrites could grow only a short distance into a groove. Migration of dendrites was not observed in this study (data not shown). As mentioned above, neurofilmanent NF-H or Tau antibodies would be useful to identify axons, and MAP2 antibody useful to identify dendrites (Kosik and Finch, 1987). Most importantly, immunocytochemical analysis clearly indicated the accumulation and migration of a single axon or a bundle of axons through microchannels (Figs. 5C" and 5D).

\section{DISCUSSION}

Microfluidics can provide a unique microenvironment, allowing precise spatial and temporal control, while monitoring cellular response, migration, and differentiation in the presence of various treatments, including cytokines, drug, etc. These unique experimental tools allow visualization of neural structure development within a microscale device. Furthermore, guidance of neurite growth, in the axonal isolation compartment in the device, can be monitored and recorded using time-lapse video microscopy (Hamon and Hong, 2013; Park et al., 2013). We have previously described a compartmental culture system of mouse embryonic stem cell (ESC)-derived neurons in microfluidic devices (Shin et al., 2010), and in the present report, have extended this approach to monitor the differentiation and migration of neural cells that are derived from hESCs. The results of the study demonstrated that microfluidics technology is a useful tool to study neurite outgrowth and axon guidance of neural cells that are derived from hESCs. In addition, the experimental approach and phenotypic outcomes using microfluidics technology are not limited to hESCs, but can be applied to various stem cell types, including patient-specific, induced pluripotent stem cells (iPSCs). A uniform population of neural cells in microfluidic devices can therefore serve as a useful tool to study pathogenesis, and can be used to screen the response of drugs to disease-specific cell lines (Bellin et al., 2012).

\section{ACKNOWLEDGMENTS}

This research was supported by grants from the Bio \& Medical Technology Development Program of the National Research Foundation (NRF) funded by the Ministry of Science, ICT \& Future Planning (2013071253), the Ministry of Food and Drug Safety in 2014 (14172MFDS974), and the Next-Generation BioGreen 21 Program (No. PJ010002012014) funded by the Rural Development Administration, Republic of Korea.

\section{REFERENCES}

Bellin, M., Marchetto, M.C., Gage, F.H., and Mummery, C.L. (2012). Induced pluripotent stem cells: the new patient? Nat. Rev. Mol. Cell. Biol. 13, 713-726

Blow, N. (2008). Stem cells: in search of common ground. Nature 451, 855-858.

Breslauer, D.N., Lee, P.J., and Lee LP. (2006). Microfluidics-based systems biology. Mol. Biosyst. 2, 97-112.

Cohen, M.S., Bas Orth, C., Kim, H.J., Jeon, N.L., and Jaffrey, S,R. (2011). Neurotrophin-mediated dendrite-to-nucleus signaling revealed by microfluidic compartmentalization of dendrites. Proc. Natl. Acad. Sci. USA 108, 11246-11251.

Hamon, M., and Hong, J.W. (2013). New tools and new biology: recent miniaturized systems for molecular and cellular biology. Mol. Cells 36, 485-506.

Jeon, I., Lee, N., Li, J.Y., Park, I.H., Park, S., Moon, J., Shim, S.H., Choi, C., Chang, D.J., Kwon, J., et al. (2012). Neural properties, in vivo effects and pathology of a Huntington's disease patientderived induced pluripotent stem cells. Stem Cells 30, 20542062.

Kim, H.J., Park, J.W., Byun, J.H., Vahidi, B., Rhee, S.W., and Jeon, N.L. (2012). Integrated microfluidics platforms for investigating injury and regeneration of CNS axons. Ann. Biomed. Eng. 40, 1268-1276

Kosik, K.S., and Finch, E.A. (1987). MAP2 and Tau segregate into dendrite and axon domains after the elaboration of morphologically distinct neuritis: an immunocytochemical study of cultured rat cerebrum. J. Neurosci. 7, 3142-3153.

Lee, N., Choi, C., Jeon, I., and Song, J. (2009). Differentiation of 
GABAergic neurons from human embryonic stem cells. Tissue Eng. Regen. Med. 6, 1359-1365.

Millet, L.J., and Gillette, M.U. (2012). New perspectives on neuronal development via microfluidic environments. Trends Neurosci. 35 752-761.

Millet, L.J., Stewart, M.E., Nuzzo, R.G., and Gillette, M.U. (2010). Guiding neuron development with planar surface gradients of substrate cues deposited using microfluidic devices. Lab. Chip $10,1525-1535$.

Park, J.W., Vahidi, B., Taylor, A.M., Rhee, S.W., and Jeon, N.L. (2006). Microfluidic culture platform for neuroscience research. Nat. Protoc. 1, 2128-2136.

Park, J.W., Kim, H.J., Byun, J.H., Ryu, H.R., and Jeon, N.L. (2009)

Novel microfluidic platform for culturing neurons: culturing and biochemical analysis of neuronal components. Biotechnol. J. 4, 1573-1577.

Park, J.W., Kim, H.J., Kang, M.W., and Jeon, N.L. (2013). Advances in microfluidics-based experimental methods for neuroscience research. Lab. Chip 13, 509-521.

Reubinoff, B.E., Pera, M.F., Fong, C.Y., Trounson, A., and Bongso, A. (2000). Embryonic stem cell lines from human blastocysts: somatic differentiation in vitro. Nat. Biotechnol. 18, 399-404.

Rhee, S.W., Taylor, A.M., Tu, C.H., Cribbs, D.H., Cotman, C.W., and Jeon, N.L. (2005). Patterned cell culture inside microfluidic devices. Lab. Chip 2005 5, 102-107.

Shin, H.S., Kim, H.J., Min, S.K., Kim, S.H., Lee, B.M., and Jeon N.L. (2010). Compartmental culture of embryonic stem cellderived neurons in microfluidic devices for use in axonal biology. Biotechnol. Lett. 32, 1063-1070.

Taylor, A.M., Rhee, S.W., and Jeon, N.L. (2006). Microfluidic chambers for cell migration and neuroscience research. Methods Mol. Biol. 321, 167-177.

Thomson, J.A., Itskovitz-Eldor, J., Shapiro, S.S., Waknitz, M.A., Swiergiel, J.J., Marshall, V.S., and Jones, J.M. (1998). Embryonic stem cell lines derived from human blastocysts. Science 282, 1145-1147. 